\title{
UPPER TRIANGULAR OPERATORS WITH SVEP
}

\author{
Bhagwati Prashad Duggal
}

\begin{abstract}
A Banach space operator $A \in B(\mathcal{X})$ is polaroid if the isolated points of the spectrum of $A$ are poles of the resolvent of $A ; A$ is hereditarily polaroid, $A \in(\mathcal{H P})$, if every part of $A$ is polaroid. Let $\mathcal{X}^{n}=\bigoplus_{i=1}^{n} \mathcal{X}_{i}$, where $\mathcal{X}_{i}$ are Banach spaces, and let $\mathcal{A}$ denote the class of upper triangular operators $A=\left(A_{i j}\right)_{1 \leq i, j \leq n}, A_{i j} \in B\left(\mathcal{X}_{j}, \mathcal{X}_{i}\right)$ and $A_{i j}=0$ for $i>j$. We prove that operators $A \in \mathcal{A}$ such that $A_{i i}$ for all $1 \leq i \leq n$, and $A^{*}$ have the single-valued extension property have spectral properties remarkably close to those of Jordan operators of order $n$ and $n$-normal operators. Operators $A \in \mathcal{A}$ such that $A_{i i} \in(\mathcal{H P})$ for all $1 \leq i \leq n$ are polaroid and have SVEP; hence they satisfy Weyl's theorem. Furthermore, $A+R$ satisfies Browder's theorem for all upper triangular operators $R$, such that $\oplus_{i=1}^{n} R_{i i}$ is a Riesz operator, which commutes with $A$.
\end{abstract}

\section{Introduction}

Let $B(\mathcal{H})$ denote the algebra of operators on a separable infinite dimensional Hilbert space $\mathcal{H}$, and let $n \in \mathbb{N}$ be a fixed natural number. An operator $A \in B(\mathcal{H})$ is a $\mathcal{C}_{n}=\mathcal{C}_{n}(\mathcal{H})$ operator if there exists a decomposition $\mathcal{H}=$ $\bigoplus_{i=1}^{n} \mathcal{H}_{i}$ and an $n \times n$ upper triangular operator matrix $A=\left(A_{i j}\right)_{1 \leq i, j \leq n}$, $A_{i j} \in B\left(\mathcal{H}_{j}, \mathcal{H}_{i}\right)$ and $A_{i j}=0$ for all $i>j$, such that $A_{i i}$ is normal for all $1 \leq i \leq n . \mathcal{C}_{n}$ operators have recently been considered by Jung, Ko, and Pearcy in [10], where it is proved that the classes consisting of Jordan operators of order $n$ and $n$-normal operators are subclasses of $\mathcal{C}_{n} . \mathcal{C}_{n}$ operators share a number of spectral properties with normal operators. More precisely, one has [10, Theorem 2.3 and Lemma 2.4]:

Theorem 1.1. If $A \in \mathcal{C}_{n}$, then $\sigma(A)=\sigma_{a}(A)=\cup_{i=1}^{n} \sigma\left(A_{i i}\right)=\sigma_{e}(A) \cup \sigma_{p}(A)$, $\sigma_{e}(A)=\cup_{i=1}^{n} \sigma_{e}\left(A_{i i}\right)=\sigma_{l e}(A) \cap \sigma_{r e}(A)=\operatorname{acc\sigma }(A) \cup \sigma_{p}^{\infty}(A)$ and $\sigma_{p}(A)$ is at most countable. Furthermore, every hole in $\sigma_{e}(A)$ has associated Fredholm index zero and intersects $\sigma(A)$ in at most countably many points.

Received April 24, 2008.

2000 Mathematics Subject Classification. Primary 47B47, 47A10, 47A11.

Key words and phrases. Banach space, $n$-normal operator, hereditarily polaroid operator, single valued extension property, Weyl's theorem. 
Here, and in the sequel, $\sigma_{a}(A)$ denotes the approximate point spectrum, $\sigma_{p}(A)$ the eigenvalues, $\sigma_{p}^{\infty}(A)$ the set of eigenvalues of infinite multiplicity, $\sigma_{l e}(A)$ and $\sigma_{r e}(A)$ the upper semi-Fredholm and the lower semi-Fredholm spectrum (respectively), $\sigma_{e}(A)$ the Fredholm spectrum, and $\operatorname{acc} \sigma(A)$ the set of accumulation points of the spectrum of $A$. Recall that a hole in $\sigma_{e}(A)$ is a bounded component of $\mathbb{C} \backslash \sigma_{e}(A)[13]$.

A part of a Banach space operator $A \in B(\mathcal{X})$ is its restriction to a closed invariant subspace. The operator $A$ is polaroid, [8], if the isolated points $\lambda$ of $\sigma(A), \lambda \in \operatorname{iso} \sigma(A)$, are poles of the resolvent of $A$ (i.e., if $\lambda \in \operatorname{iso} \sigma(A)$ implies $\operatorname{asc}(A-\lambda)=\operatorname{dsc}(A-\lambda)<\infty$, where the ascent $\operatorname{asc}(A-\lambda)$ of $A-\lambda$ is the least non-negative integer $n$ such that $(A-\lambda)^{-n}(0)=(A-\lambda)^{(n+1)}(0)$ and the descent $\operatorname{dsc}(A-\lambda)$ of $A-\lambda$ is the least non-negative integer $n$ such that $\left.(A-\lambda)^{n} \mathcal{X}=(A-\lambda)^{n+1} \mathcal{X}\right)$. We say that $A \in B(\mathcal{X})$ is hereditarily polaroid, $A \in(\mathcal{H P})$, if every part of $A$ is polaroid [6]. Class $(\mathcal{H} \mathcal{P})$ is large: it contains a number of the more commonly considered classes of operators, amongst them subscalar operators, multipliers of commutative semi-simple Banach algebras and paranormal operators (See [6] for an extensive, though by no means exhaustive, list of classes of operators which are $(\mathcal{H} \mathcal{P})$ operators). Just like normal operators, $(\mathcal{H P})$ operators have SVEP, the single-valued extension property [6, Theorem 2.8]; however, unlike normal operators, the conjugate operator $A^{*}$ of an operator $A \in(\mathcal{H} \mathcal{P})$ does not (necessarily) have SVEP.

Let $\mathcal{X}^{n}=\bigoplus_{i=1}^{n} \mathcal{X}_{i}$, where each $\mathcal{X}_{i}, 1 \leq i \leq n$, is an infinite dimensional Banach space. Let $\mathcal{A}$ denote the class of upper triangular operator matrices

$$
A=\left(A_{i j}\right)_{1 \leq i, j \leq n} \in B\left(\mathcal{X}^{n}\right), \quad A_{i j} \in B\left(\mathcal{X}_{j}, \mathcal{X}_{i}\right) \text { and } A_{i j}=0 \text { for all } i>j,
$$

and let $(H P)_{n}$ denote the class of operators $A \in \mathcal{A}$ such that $A_{i i} \in(\mathcal{H P})$ for all $1 \leq i \leq n$. We study operators $A \in(H P)_{n}$, and operators $A \in \mathcal{A}$ such that $A_{i i}$ has SVEP for all $1 \leq i \leq n$. It is proved that operators $A \in \mathcal{A}$ such that each $A_{i i}$ has SVEP have spectral properties remarkably similar to those of operators $A \in \mathcal{C}_{n}$. Indeed, if $A \in \mathcal{A}$ is such that $A^{*}$ along with each $A_{i i}$ has SVEP, then $A$ shares with operators in $\mathcal{C}_{n}$ the spectral properties of Theorem 1.1 (except for the countability properties). We prove that if the Banach spaces $\mathcal{X}_{i}$ are separable and the operators $A_{i i}$ are hereditarily normaloid $(\mathcal{H} \mathcal{P})$ operators (see the following sections for any undefined terminology), then $\sigma_{p}(A)$ is at most countable. Operators $A \in(H P)_{n}$ are polaroid and have SVEP. It is proved that if $A \in \mathcal{A}$ and $A_{0}=\bigoplus_{i=1}^{n} A_{i i}$ is a polynomially $(H P)_{n}$ operator which commutes with the diagonal algebraic operator $B=\bigoplus_{i=1}^{n} B_{i i} \in B\left(\mathcal{X}^{n}\right)$, then $f(A+B)$ satisfies Weyl's theorem and $f\left(A^{*}+B^{*}\right)$ satisfies $a$-Weyl's theorem for every function $f$ which is analytic on a neighbourhood of $\sigma(A+B)$. Perturbation of operators $A \in \mathcal{A}$ such that $A_{i i}$ has SVEP for all $1 \leq i \leq n$ by quasi-nilpotent operators is considered.

\section{Upper triangular operators with SVEP}

An operator $A \in B(\mathcal{X})$ is upper semi-Fredholm (lower semi-Fredholm) at 
$\lambda \in \mathbb{C}, \lambda \in \Phi_{+}(A)$ (resp., $\left.\lambda \in \Phi_{-}(A)\right)$, if $(A-\lambda) \mathcal{X}$ is closed and $\alpha(A-\lambda)=$ $\operatorname{dim}(A-\lambda)^{-1}(0)<\infty$ (resp., $(A-\lambda) \mathcal{X}$ is closed and $\beta(A-\lambda)=\operatorname{dim}(\mathcal{X} \backslash$ $(A-\lambda) \mathcal{X})<\infty)$. The upper semi-Fredholm spectrum (resp., lower semiFredholm spectrum) of $A$ is the set $\sigma_{l e}(A)=\left\{\lambda \in \mathbb{C}: \lambda \notin \Phi_{+}(A)\right\}$ (resp., $\left.\sigma_{r e}(A)=\left\{\lambda \in \mathbb{C}: \lambda \notin \Phi_{-}(A)\right\}\right)$, the semi-Fredholm spectrum of $A$ is the set $\sigma_{s F}(A)=\sigma_{l e}(A) \cap \sigma_{r e}(A)$, and the Fredholm spectrum of $A$ is the set $\sigma_{e}(A)=\sigma_{l e}(A) \cup \sigma_{r e}(A)=\left\{\lambda \in \sigma(A): \lambda \notin \Phi(A)=\Phi_{+}(A) \cap \Phi_{-}(A)\right\}$. The Weyl spectrum (resp., Browder spectrum) of $A$ is the set $\sigma_{w}(A)=\{\lambda \in \mathbb{C}: A-\lambda$ is not Fredholm or ind $(A-\lambda) \neq 0\}$ (resp., $\sigma_{b}(A)=\{\lambda \in \mathbb{C}: A-\lambda$ is not Fredholm or $\operatorname{asc}(A-\lambda) \neq \operatorname{dsc}(A-\lambda)\}$. Here ind $(A-\lambda)=\alpha(A-\lambda)-\beta(A-\lambda)$ is the Fredholm index of $A-\lambda$. Let $\pi_{00}(A)=\{\lambda \in \operatorname{iso} \sigma(A): 0<\alpha(A-\lambda)<\infty\}$, and let $p_{0}(A)=\{\lambda \in \operatorname{iso} \sigma(A): \lambda$ is a finite rank pole of the resolvent of $A\}$. In common with current terminology, we say that $A$ satisfies Weyl's theorem, shortened to $A$ satisfies $W t$, if $\sigma(A) \backslash \sigma_{w}(A)=\pi_{00}(A) ; A$ satisfies Browder's theorem, or $B t$, if $\sigma_{w}(A)=\sigma_{b}(A)\left(\Longleftrightarrow \sigma(A) \backslash \sigma_{w}(A)=p_{0}(A)\right)$.

An operator $A \in B(\mathcal{X})$ has the single-valued extension property at a point $\lambda_{0} \in \mathbb{C}$, SVEP at $\lambda_{0}$, if for every open $\operatorname{disc} \mathcal{D}$ centered at $\lambda_{0}$ the only analytic function $f: \mathcal{D} \longrightarrow \mathcal{X}$ satisfying $(A-\lambda) f(\lambda)=0$ is the function $f \equiv 0$. The single valued extension property plays an important role in local spectral theory and Fredholm theory (see [11] and [1]). Evidently, every $A$ has SVEP at points in the resolvent set $\rho(A)=\mathbb{C} \backslash \sigma(A)$ and the boundary $\partial \sigma(A)$ of $\sigma(A)$. It is easily verified that SVEP is inherited by restrictions. We say that $A$ has SVEP if it has SVEP at every $\lambda \in \sigma(A)$.

Lemma 2.1. If $\lambda \in \Phi_{ \pm}(A)$, then the following implications hold:

$A$ (resp., $\left.A^{*}\right)$ has SVEP at $\lambda \Longrightarrow \operatorname{asc}(A-\lambda)<\infty \quad($ resp., dsc $(A-\lambda)<\infty)$;

$\operatorname{asc}(A-\lambda)<\infty \Longrightarrow \operatorname{ind}(A-\lambda) \leq 0 \quad($ resp., $\operatorname{dsc}(A-\lambda)<\infty \Longrightarrow \operatorname{ind}(A-\lambda) \geq 0)$.

Proof. See [1, Theorems 3.16, 3.17 and Corollary 3.19].

The following theorem is known (see, for example, [2, Lemma 2.18] and [3, Theorem 2.2]).

Theorem 2.2. Let $A \in B(\mathcal{X})$.

(i) A necessary and sufficient condition for A to satisfy Browder's theorem, $\sigma(A) \backslash \sigma_{w}(A)=p_{0}(A)$ (equivalently, $\sigma_{b}(A)=\sigma_{w}(A)$ ), is that $A$ has SVEP at points $\lambda \notin \sigma_{w}(A)$.

(ii) A necessary and sufficient condition for A to satisfy Weyl's theorem is that $A$ satisfies Browder's theorem and $A$ is polaroid at points $\lambda \in \pi_{00}(A)$.

It is well known that $A$ satisfies Browder's theorem if and only if $A^{*}$ satisfies Browder's theorem: this, however, fails for Weyl's theorem.

The quasinilpotent part $H_{0}(A)$ and the analytic core $K(A)$ of an operator $A \in B(\mathcal{X})$ are the sets

$$
H_{0}(A)=\left\{x \in \mathcal{X}: \lim _{n \longrightarrow \infty}\left\|A^{n} x\right\|^{\frac{1}{n}}=0\right\}
$$


and

$K(A)=\left\{x \in \mathcal{X}:\right.$ there exists a sequence $\left\{x_{n}\right\} \subset \mathcal{X}$ and $\delta>0$ for which $x=x_{0}, A x_{n+1}=x_{n}$ and $\left\|x_{n}\right\| \leq \delta^{n}\|x\|$ for all $\left.n \in \mathbb{N}\right\}$

[1, 11]. $H_{0}(A)$ and $K(A)$ are generally non-closed hyperinvariant subspaces of $A$ such that $A^{-m}(0) \subseteq H_{0}(A)$ for all $m \in \mathbb{N}$ and $A K(A)=K(A)$ [12]. If $\lambda \in \operatorname{iso} \sigma(A)$, then

$$
\mathcal{X}=H_{0}(A-\lambda) \oplus K(A-\lambda)
$$

[1, Theorem 3.74]; furthermore, if also $\lambda \in \Phi_{ \pm}(A)$, then, [1, Theorem 3.77], $H_{0}(A-\lambda)=(A-\lambda)^{-p}(0)$ for some $p \in \mathbb{N}$ and

$$
\mathcal{X}=(A-\lambda)^{-p}(0) \oplus(A-\lambda)^{p} \mathcal{X}
$$

(so that $\lambda$ is a finite rank pole of the resolvent of $A$ ).

Operators $A \in(\mathcal{H P})$ have SVEP [6, Theorem 2.8], and SVEP survives quasi-affine transforms. Indeed, if $S X=X T$, where $S \in B(\mathcal{X}), X \in B(\mathcal{Y}, \mathcal{X})$ is injective and $T \in B(\mathcal{Y})$, then $(T-\lambda) f(\lambda)=0 \Longrightarrow(S-\lambda) X f(\lambda)=0$ : hence if $S$ has SVEP at a point $\lambda_{0}$ and $\lambda$ is a point in an open disc centered at $\lambda_{0}$, then $X f(\lambda) \equiv 0 \Longrightarrow f(\lambda) \equiv 0$. The polaroid property survives similarities, but it does not survive quasi-affine transforms, as the following example shows. Let $T \in \ell^{2}(\mathbb{N})$ be the forward unilateral shift, let $S \in \ell^{2}(\mathbb{N})$ be the weighted forward unilateral shift with the weight sequence $\left\{\frac{1}{n+1}\right\}$, and let $X \in \ell^{2}(\mathbb{N})$ be the multiplication operator defined by $X x=\left\{\frac{x_{n}}{n !}\right\}_{n \in \mathbb{N}}$ for all $x=\left\{x_{n}\right\}_{n \in \mathbb{N}} \in \ell^{2}(\mathbb{N})$. Then $T \in(\mathcal{H P}), X$ is a quasi-affinity and $S X=X T$, but $S$ is quasi-nilpotent (hence not polaroid).

Operators $A \in \mathcal{A}$ such that $A_{i i}$ has SVEP for all $1 \leq i \leq n$ have SVEP [4, Lemma 3.1]. Furthermore:

Theorem 2.3. If $A \in \mathcal{A}$ is such that $A_{i i}$ has $S V E P$ for all $1 \leq i \leq n$, then:

(i) $\sigma(A)=\sigma_{a}\left(A^{*}\right)=\bigcup_{i=1}^{n} \sigma_{a}\left(A_{i i}^{*}\right)=\bigcup_{i=1}^{n} \sigma\left(A_{i i}\right)=\sigma_{w}(A) \cup \pi_{00}(A)=$ $\sigma_{b}(A) \cup \pi_{00}(A)$

(ii) $\sigma_{e}(A)=\sigma_{r e}(A)=\bigcup_{i=1}^{n} \sigma_{r e}\left(A_{i i}\right)=\bigcup_{i=1}^{n} \sigma_{e}\left(A_{i i}\right)$;

(iii) $\sigma_{s F}(A)=\sigma_{l e}(A)=a c c \sigma_{a}(A) \cup\left\{\lambda \in i s o \sigma_{a}(A): \operatorname{dim} H_{0}(A-\lambda)=\infty\right.$ or $(A-\lambda) \mathcal{X}^{n}$ is not closed\};

(iv) $\sigma_{w}(A)=\bigcup_{i=1}^{n} \sigma_{w}\left(A_{i i}\right)$, and $\sigma_{w}(A)=\sigma_{e}(A)$ if and only if $A^{*}$ has $S V E P$ at points $\lambda \in \sigma_{w}(A) \backslash \sigma_{e}(A)$;

(v) if $C$ is a hole in $\sigma_{e}(A)$, then, for all $\lambda \in C$, either ind $(A-\lambda)=0$ and $C \cap \rho(A) \neq \emptyset$ or ind $(A-\lambda)<0$ and the eigenvalues do not have a limit point in $C$ (and every point of $C$ is a deficiency value).

Furthermore, if also $A^{*}$ has SVEP, then:

(vi) $\sigma_{e}(A)=\sigma_{l e}(A) \cap \sigma_{r e}(A)=\sigma_{s F}(A)=\sigma_{w}(A)$ and $\sigma(A)=\sigma_{a}(A)=$ $\sigma_{e}(A) \cup \pi_{00}(A)$

(vii) for every hole $C$ in $\sigma_{e}(A)$ and $\lambda \in C$, ind $(A-\lambda)=0$ and $C \cap \rho(A) \neq \emptyset$. 
Proof. A proof of parts (i) to (iv) of the theorem, except for the equality $\sigma(A)=\sigma_{w}(A) \cup \pi_{00}(A)=\sigma_{b}(A) \cup \pi_{00}(A)$ of part (i), the equality $\sigma_{s F}(A)=$ $\sigma_{l e}(A)$ of part (iii), and the necessary and sufficient condition for the equality of the spectra $\sigma_{w}(A)$ and $\sigma_{e}(A)$ of part (iv), appears in [4, Proposition 3.5]. We divide the proof into parts $(\mathbf{A})$ and $(\mathbf{B})$, with $(\mathbf{A})$ completing the proof of parts (i) to (v), and (B) proving parts (vi) and (vii).

(A). Start by observing that $A$ has SVEP implies $\sigma_{w}(A)=\sigma_{b}(A)$. Evidently, $\sigma_{b}(A) \cup \pi_{00}(A) \subseteq \sigma(A)$. (Recall that $\pi_{00}(A)=\{\lambda \in \operatorname{iso} \sigma(A): 0<$ $\alpha(A-\lambda)<\infty\}$.) For the reverse inclusion, if $\lambda \in \sigma(A) \backslash \sigma_{b}(A)$, then $\lambda \in$ $\Phi(A)=\Phi_{+}(A) \cap \Phi_{-}(A)$ and $\operatorname{asc}(A-\lambda)=\operatorname{dsc}(A-\lambda)<\infty$. Hence $\lambda$ is a finite rank pole of the resolvent of $A$. Thus $\lambda \in \pi_{00}(A)$ and $\sigma(A) \subseteq \sigma_{b}(A) \cup \pi_{00}(A)$. To complete the proof of (iii), let $\lambda \notin \sigma_{r e}(A)$. Then $\lambda \in \Phi_{-}(A)$. Since $A$ has $\mathrm{SVEP}$, ind $(A-\lambda)=\alpha(A-\lambda)-\beta(A-\lambda) \leq 0$. Thus $\lambda \notin \sigma_{r e}(A) \Longrightarrow(A-\lambda) \mathcal{X}^{n}$ is closed and $\alpha(A-\lambda) \leq \beta(A-\lambda)<\infty$. Hence $\lambda \notin \sigma_{l e}(A)$, which implies that $\sigma_{l e}(A) \subseteq \sigma_{r e}(A)$. Thus $\sigma_{s F}(A)=\sigma_{l e}(A) \cap \sigma_{r e}(A)=\sigma_{l e}(A)$.

To complete the proof of (iv), we start by observing that $A$ has SVEP implies $A$ satisfies Browder's theorem, which in turn implies that $A^{*}$ satisfies Browder's theorem, i.e., $A^{*}$ has SVEP at $\lambda \notin \sigma_{w}\left(A^{*}\right)=\sigma_{w}(A)$. Thus if $\sigma_{w}(A)=\sigma_{e}(A)$, then (vacuously) $A^{*}$ has SVEP at points $\lambda \in \sigma_{w}(A) \backslash \sigma_{e}(A)$. Conversely, if $A^{*}$ has SVEP at points $\lambda \in \sigma_{w}(A) \backslash \sigma_{e}(A)$, then $A^{*}$ has SVEP at all $\lambda \in \Phi\left(A^{*}\right)=\Phi(A)$. This, since $A$ has SVEP, implies that if $\lambda \in \Phi(A)$, then $\operatorname{ind}(A-\lambda)=0$. Hence $\sigma_{w}(A) \subseteq \sigma_{e}(A)$. Since $\sigma_{e}(A) \subseteq \sigma_{w}(A)$ for every $A, \sigma_{w}(A)=\sigma_{e}(A)$.

To prove (v), we observe that if $C$ is a hole in $\sigma_{e}(A)$ and $\lambda \in C$, then $\lambda \in \Phi(A)$ and (because of $\operatorname{SVEP}) \operatorname{ind}(A-\lambda) \leq 0$. If $\operatorname{ind}(A-\lambda)=0$, then (both $A$ and $A^{*}$ have SVEP at $\lambda$ and) $\operatorname{asc}(A-\lambda)=\operatorname{dsc}(A-\lambda)<\infty$; if, instead, $\operatorname{ind}(A-\lambda)<0$, then ( $A$ has SVEP at $\lambda$ implies) $\operatorname{asc}(A-\lambda)<\infty$. Now apply [9, Theorem $\left.51.1\left(a_{1}\right),\left(b_{1}\right)\right]$.

(B). Observe that if $A^{*}$ has SVEP, then $\sigma(A)=\sigma_{a}(A)$ [11, Proposition 1.3.2]. Thus, if both $A$ and $A^{*}$ have SVEP, then (see (iv)) $\sigma(A)=\sigma_{a}(A)=$ $\sigma_{e}(A) \cup \pi_{00}(A)$. Again, if $A^{*}$ has SVEP, then $\lambda \notin \sigma_{l e}(A)$ implies that $\lambda \in \Phi_{+}(A)$ and $\operatorname{ind}(A-\lambda) \geq 0 \Longrightarrow \lambda \in \Phi(A)$ (and $\operatorname{ind}(A-\lambda) \geq 0)$. Thus $\lambda \notin \sigma_{r e}(A)$, which in view of the fact that $\sigma_{l e}(A) \subseteq \sigma_{r e}(A)$, see the proof above, implies that $\sigma_{l e}(A)=\sigma_{r e}(A)$. This proves (vi). The proof of (vii) follows from $[9$, Theorem 51.1].

It is apparent from the theorem above that a number of the properties of operators $A \in \mathcal{C}_{n}$ proved in Theorem 1.1 are typical of operators $A \in \mathcal{A}$ such that $A_{i i}, 1 \leq i \leq n$, and $A^{*}$ have SVEP. The following theorem supplements Theorem 2.3 to prove that if $\mathcal{X}$ is separable and $A \in \mathcal{A}$, then $\sigma_{p}(A)$ is countable for certain classes of operators $A_{i i}$.

Recall that an operator $T \in B(\mathcal{X})$ is normaloid if its norm equals its spectral radius. We say that $T$ is hereditarily normaloid if every part of $T$ is normaloid. 
We shall call an operator simply polaroid if the isolated points of its spectrum are simple poles, i.e., poles of order one, of its resolvent.

Theorem 2.4. Let $\mathcal{X}$ be a separable Banach space, and let $T \in B(\mathcal{X})$ be a hereditarily normaloid $(\mathcal{H} \mathcal{P})$ operator. Then $\sigma_{p}(T)$ is countable.

Proof. Let $\alpha, \beta \in \sigma_{p}(T)$, where $\beta \neq 0, \alpha \neq \beta$ and $|\alpha| \leq|\beta|$. Let $M$ denote the subspace generated by $(T-\alpha)^{-1}(0)$ and $(T-\beta)^{-1}(0)$. Then $T_{1}=\left.T\right|_{M}$ is a normaloid $(\mathcal{H P})$ operator with $\sigma\left(T_{1}\right)=\{\alpha, \beta\}$, and $\left\|T_{1}\right\|=|\beta|$. Furthermore, $\operatorname{asc}\left(T_{1}-\beta\right) \leq 1[9$, Proposition 54.2], which implies that $\beta$ is a simple pole of the resolvent of $T_{1}$. Let $P_{\beta}$ denote the spectral projection corresponding to $\beta$; then $\left\|P_{\beta}\right\|=1[9$, Proposition 54.4]. Since

$M=P_{\beta} M \oplus\left(1-P_{\beta}\right) M=\left(T_{1}-\beta\right)^{-1}(0) \oplus\left(T_{1}-\beta\right) M=(T-\beta)^{-1}(0) \oplus(T-\alpha)^{-1}(0)$,

it follows that

$$
\|x\|=\left\|P_{\beta} x\right\|=\left\|P_{\beta}(x+y)\right\| \leq\|x+y\|
$$

for every $x \in(T-\beta)^{-1}(0)$ and $y \in\left(T_{1}-\beta\right) M=(T-\alpha)^{-1}(0)$. Suppose now that $\sigma_{p}(T)$ is not countable. Then there exists an uncountable set of unit vectors $\left\{x_{i}\right\}$ and $\left\{y_{i}\right\}$, determined by the unit eigen-vectors corresponding to distinct eigen-values of $T$ such that

$$
1 \leq\left\|x_{i}+y_{i}\right\|
$$

Since $\mathcal{X}$ is separable, this is impossible. Hence $\sigma_{p}(T)$ is countable.

Theorem 2.4 implies that if $\mathcal{X}$ is separable, each $A_{i i}, 1 \leq i \leq n$, is a hereditarily normaloid $(\mathcal{H} \mathcal{P})$ operator and $A$ is the upper triangular operator $A=\left(A_{i j}\right)_{1 \leq i, j \leq n}$, then $\sigma_{p}(A)$ is at most countable. The hypotheses of Theorem 2.4 are satisfied by a number of classes of Banach space operators, amongst them $\mathcal{T H} \mathcal{N}$ and $\mathcal{C H N}$ operators of [2]. Suffice it to say here that paranormal operators, [9, page 229], are operators of the type considered in the theorem.

Perturbations. An operator $R \in B(\mathcal{X})$ is a Riesz operator if $\lambda \in \Phi(R)$ for every non-zero $\lambda \in \mathbb{C}$ : equivalently, $R$ is a Riesz operator if the essential numerical radius $r_{e}(R)=\lim _{n \longrightarrow \infty}\left\|\pi(R)^{n}\right\|^{\frac{1}{n}}=0$, where $\pi: B(\mathcal{X}) \longrightarrow B(\mathcal{X}) / \mathcal{K}(\mathcal{X})$ is the Calkin map and $\mathcal{K}(\mathcal{X}) \subset B(\mathcal{X})$ is the ideal of compact operators. Evidently, quasi-nilpotent operators are Riesz operators. The following theorem, the main result of this section, considers perturbations of operators $A \in \mathcal{A}$ such that $A_{i i}$ has SVEP for all $1 \leq i \leq n$ by upper triangular operators $R$ such that $[A, R]=0$ and $R_{0}=\oplus_{i=1}^{n} R_{i i}$ is a Riesz operator. But before that we introduce some terminology and complementary results specific to this and the following results.

For an operator $T \in B(\mathcal{X})$, let $T \in \Phi(\mathcal{X})$ (resp., $T \in \Phi_{+}(\mathcal{X}), T \in \Phi_{-}(\mathcal{X})$ ) denote that $T$ is Fredholm (resp., upper semi-Fredholm, lower semi-Fredholm). 
The Weyl essential approximate point spectrum $\sigma_{w a}(T)$ and the Weyl essential surjectivity spectrum $\sigma_{w s}(T)$ of $T \in B(\mathcal{X})$ are the sets

$\sigma_{w a}(T)=\bigcap_{K \in \mathcal{K}(\mathcal{X})} \sigma_{a}(T+K)=\left\{\lambda \in \sigma_{a}(T): T-\lambda \notin \Phi_{+}(\mathcal{X})\right.$ or $\left.\operatorname{ind}(T-\lambda) \not \leq 0\right\}$ and

$\sigma_{w s}(T)=\bigcap_{K \in \mathcal{K}(\mathcal{X})} \sigma_{s}(T+K)=\left\{\lambda \in \sigma_{s}(T): T-\lambda \notin \Phi_{-}(\mathcal{X})\right.$ or ind $\left.(T-\lambda) \nsupseteq 0\right\}$.

Evidently, $\sigma_{w s}(T)=\sigma_{w a}\left(T^{*}\right), \sigma_{w a}(T)=\sigma_{w s}\left(T^{*}\right)$ and $\sigma_{w}(T)=\sigma_{w a}(T) \cup$ $\sigma_{w s}(T)$. The Browder essential approximate point spectrum $\sigma_{b a}(T)$ and the Browder essential surjectivity spectrum $\sigma_{b s}(T)$ of $T \in B(\mathcal{X})$ are the sets

$\sigma_{b a}(T)=\bigcap_{K \in \mathcal{L}(\mathcal{X}), K T=T K} \sigma_{a}(T+K)=\left\{\lambda \in \sigma_{a}(T): T-\lambda \notin \Phi_{+}(\mathcal{X})\right.$ or $\left.\operatorname{asc}(T-\lambda)=\infty\right\}$

and

$\sigma_{b s}(T)=\bigcap_{K \in \kappa(\mathcal{X}), K T=T K} \sigma_{s}(T+K)=\left\{\lambda \in \sigma_{s}(T): T-\lambda \notin \Phi_{-}(\mathcal{X}) \operatorname{or} \operatorname{dsc}(T-\lambda)=\infty\right\}$.

Apparently, $\sigma_{b s}(T)=\sigma_{b a}\left(T^{*}\right), \sigma_{b a}(T)=\sigma_{b s}\left(T^{*}\right), \sigma_{b}(T)=\sigma_{b a}(T) \cup \sigma_{b s}(T)$, $\sigma_{w a}(T) \subseteq \sigma_{b a}(T)$ and $\sigma_{w s}(T) \subseteq \sigma_{b s}(T)$. We say that $T$ satisfies $a$-Browder's theorem, or $a-B t$ (resp., $s$-Browder's theorem, or $s-B t$ ) if $\sigma_{w a}(T)=\sigma_{b a}(T)$ (resp., $\sigma_{w s}(T)=\sigma_{b s}(T)$ ).

Lemma 2.5. $T$ satisfies $a-B t$ (rep., $s-B t)$ if and only $T$ has $S V E P$ at points $\lambda \notin \sigma_{w a}(T)$ (resp., $\left.\lambda \notin \sigma_{w s}(T)\right)$.

Proof. The lemma is proved in [2, Lemma 2.18] for the case in which $T$ satisfies $a-B t$; the case in which $T$ satisfies $s-B t$ is similarly proved.

Lemma 2.6. If $T$ has $S V E P$, then $T$ and $T^{*}$ satisfy $a-B t$ and $s-B t$.

Proof. It is clear from Lemma 2.5 that $T$ satisfies $a-B t$ and $s-B t$. To prove that $T^{*}$ satisfies $a-B t$ and $s-B t$, we have only to prove that $\sigma_{b a}\left(T^{*}\right) \subseteq$ $\sigma_{w a}\left(T^{*}\right)$ and $\sigma_{b s}\left(T^{*}\right) \subseteq \sigma_{w s}\left(T^{*}\right)$. Observe that if $\lambda \notin \sigma_{w a}\left(T^{*}\right)$ (resp., $\lambda \notin$ $\left.\sigma_{w s}\left(T^{*}\right)\right)$, then $T^{*}-\lambda I^{*} \in \Phi_{+}\left(\mathcal{X}^{*}\right)$ and $\operatorname{ind}\left(T^{*}-\lambda I^{*}\right) \leq 0$ (resp., $T^{*}-\lambda I^{*} \in$ $\Phi_{-}\left(\mathcal{X}^{*}\right)$ and $\operatorname{ind}\left(T^{*}-\lambda I^{*}\right) \geq 0$ ). Thus, if $T$ has SVEP and $\lambda \notin \sigma_{w a}\left(T^{*}\right)$ (resp., $\left.\lambda \notin \sigma_{w s}\left(T^{*}\right)\right)$, then, by Lemma $2.1, T^{*}-\lambda I^{*} \in \Phi\left(\mathcal{X}^{*}\right)$ and $\operatorname{asc}\left(T^{*}-\lambda I^{*}\right)=$ $\operatorname{dsc}\left(T^{*}-\lambda I^{*}\right)<\infty\left(\right.$ resp., $T^{*}-\lambda I^{*} \in \Phi\left(\mathcal{X}^{*}\right)$ and $\left.\operatorname{dsc}\left(T^{*}-\lambda I^{*}\right)<\infty\right)$. Hence $\lambda \notin \sigma_{b a}\left(T^{*}\right)$ (resp., $\left.\lambda \notin \sigma_{b s}\left(T^{*}\right)\right)$.

The following lemma follows from [14, Theorem 5] and the argument of the proof of [5, Theorem 1.5(iii)].

Lemma 2.7. If $R \in B(\mathcal{X})$ is a Riesz operator such that $[T, R]=0$, then $\sigma_{w x}(T+R)=\sigma_{w x}(T)$ and $\sigma_{b x}(T+R)=\sigma_{b x}(T)$, where $\sigma_{. x}$ stands for $\sigma_{. a}$ or $\sigma_{. s}$. 
Taken together, Lemmas 2.6 and 2.7 imply that if $T$ has SVEP and the Riesz operator $R$ commutes with $T$, then $T+R$ and $T^{*}+R^{*}$ satisfy $a-B t$ and $s-B t$. Combining with Lemma 2.5, this implies the following.

Corollary 2.8. If $T$ has SVEP and the Riesz operator $R$ commutes with $T$, then $T+R$ has SVEP at points $\lambda \notin \sigma_{w x}(T+R)$ and $T^{*}+R^{*}$ has SVEP at points $\lambda \notin \sigma_{w x}\left(T^{*}+R^{*}\right)$, where $\sigma_{. x}$ stands for $\sigma_{. a}$ or $\sigma_{. s}$.

Theorem 2.9. Let $A \in \mathcal{A}$ be such that $A_{i i}$ has $S V E P$ for all $1 \leq i \leq n$. Let $R=\left(R_{i j}\right)_{1 \leq i, j \leq n} \in B\left(\mathcal{X}^{n}\right), R_{i j}=0$ for all $i>j$, be an upper triangular operator such that $[T, R]=0$.

(i) If $R_{0}=\oplus_{i=1}^{n} R_{i i}$ is a Riesz operator, then $\sigma(A+R) \backslash \sigma_{w}(A+R)=$ $p_{0}(A+R)$.

(ii) If $R_{0}=\oplus_{i=1}^{n} R_{i i}$ is a quasi-nilpotent operator, then $A+R$ satisfies conclusions (i) to (v) of Theorem 2.3.

(iii) If $R_{0}=\oplus_{i=1}^{n} R_{i i}$ is an injective quasi-nilpotent operator, then $(A+R$ satisfies conclusions (i) to $(\mathrm{v})$ of Theorem 2.3 and $) \sigma(A+R)=\sigma_{w}(A+$ $R)=\sigma_{b}(A+R)$.

Proof. The hypothesis $R$ commutes with $A$ implies that $R_{i i}$ commutes with $A_{i i}$ for all $1 \leq i \leq n$.

(i) Since the restriction of a Riesz operator to an invariant subspace is again a Riesz operator, the hypotheses imply that $R_{i i}$ is a Riesz operator such that $\left[A_{i i}, R_{i i}\right]=0, \sigma_{w}\left(A_{i i}+R_{i i}\right)=\sigma_{w}\left(A_{i i}\right), \sigma_{b}\left(A_{i i}+R_{i i}\right)=\sigma_{b}\left(A_{i i}\right)$ for all $1 \leq i \leq n$. Furthermore, $A$ has SVEP (implies $A$ satisfies $B t$, i.e., $\left.\sigma_{w}(A)=\sigma_{b}(A)\right), \sigma_{w}(A)=\bigcup_{i=1}^{n} \sigma_{w}\left(A_{i i}\right)$ and $\sigma_{b}(A)=\bigcup_{i=1}^{n} \sigma_{b}\left(A_{i i}\right)$. Hence $\sigma_{w}(A)=\bigcup_{i=1}^{n} \sigma_{w}\left(A_{i i}+R_{i i}\right)=\bigcup_{i=1}^{n} \sigma_{b}\left(A_{i i}+R_{i i}\right)=\sigma_{b}(A)$. Evidently, $\sigma_{w}(A+$ $R) \subseteq \bigcup_{i=1}^{n} \sigma_{w}\left(A_{i i}+R_{i i}\right)$ and $\sigma_{b}(A+R) \subseteq \bigcup_{i=1}^{n} \sigma_{b}\left(A_{i i}+R_{i i}\right)$. We prove the reverse inclusions: this would then imply that $A+R$ satisfies $B t$.

Define the operator $T_{s} \in B\left(\oplus_{i=s}^{n} \mathcal{X}_{i}\right), 1 \leq s \leq n$, by

$$
T_{s}=\left(\begin{array}{clcc}
A_{s s}+R_{s s} & A_{s(s+1)}+R_{s(s+1)} & \cdots & A_{s n}+R_{s n} \\
0 & A_{(s+1)(s+1)}+R_{(s+1)(s+1)} & \cdots & A_{(s+1) n}+R_{(s+1) n} \\
\vdots & & & \\
0 & 0 & \cdots & A_{n n}+R_{n n}
\end{array}\right) .
$$

Considering the operator

$$
A+R=\left(\begin{array}{cc}
A_{11}+R_{11} & * \\
0 & T_{2}
\end{array}\right),
$$

it follows that if $\lambda \notin \sigma_{w}(A+R)$, then $A_{11}+R_{11}-\lambda \in \Phi_{+}\left(\mathcal{X}_{1}\right), T_{2}-\lambda \in$ $\Phi_{-}\left(\oplus_{i=2}^{n} \mathcal{X}_{i}\right)$ and ind $\left(A_{11}+R_{11}-\lambda\right)+\operatorname{ind}\left(T_{2}-\lambda\right)=0$. Recall from Corollary 3.2 that $A_{11}+R_{11}$ has SVEP at points $\lambda \notin \sigma_{w a}\left(A_{11}+R_{11}\right)$ and $A_{11}^{*}+R_{11}^{*}$ has $\mathrm{SVEP}$ at points $\lambda \notin \sigma_{w s}\left(A_{11}^{*}+R_{11}^{*}\right)=\sigma_{w a}\left(A_{11}+R_{11}\right)$. Hence, see Lemma 2.1, if $A_{11}+R_{11}-\lambda \in \Phi_{+}\left(\mathcal{X}_{1}\right)$ and if either $\operatorname{ind}\left(A_{11}+R_{11}-\lambda\right) \geq 0$ or $\operatorname{ind}\left(A_{11}+\right.$ $\left.R_{11}-\lambda\right) \leq 0$, then $\operatorname{ind}\left(A_{11}+R_{11}-\lambda\right)=0$ and $A_{11}+R_{11}-\lambda \in \Phi\left(\mathcal{X}_{1}\right)$ (i.e., 
$\left.\lambda \notin \sigma_{w}\left(A_{11}+R_{11}\right)\right)$. Consequently, $T_{2}-\lambda \in \Phi_{-}\left(\oplus_{i=2}^{n} \mathcal{X}_{i}\right)$ and $\operatorname{ind}\left(T_{2}-\lambda\right)=0$, i.e., $T_{2}-\lambda \in \Phi\left(\oplus_{i=2}^{n} \mathcal{X}_{i}\right)$. Repeating this argument, successively considering $T_{2}, T_{3}$ etc., it follows that if $\lambda \notin \sigma_{w}(A+R)$, then $\lambda \notin \sigma_{w}\left(A_{i i}+R_{i i}\right)$ for all $1 \leq i \leq n$. Hence $\bigcup_{i=1}^{n} \sigma_{w}\left(A_{i i}+R_{i i}\right) \subseteq \sigma_{w}(A+R)$. A similar argument proves that $\bigcup_{i=1}^{n} \sigma_{b}\left(A_{i i}+R_{i i}\right) \subseteq \sigma_{b}(A+R)$.

(ii) The argument in this case is straightforward. Since $R_{i i}$ is a quasinilpotent which commutes with $A_{i i}$ for all $1 \leq i \leq n, A_{i i}$ and $A_{i i}+R_{i i}$ are quasinilpotent equivalent $\left[11\right.$, p. 253]. Since $A_{i i}$ has SVEP for all $1 \leq i \leq n, A_{i i}+R_{i i}$ has SVEP [11, Proposition 3.4.1]. Hence $A+R$ has SVEP (so that Theorem 2.3 applies).

(iii) Evidently, $A_{i i}+R_{i i}$ has SVEP for all $1 \leq i \leq n$, Theorem 2.3 applies and we conclude that $\sigma_{w}(A+R)=\sigma_{b}(A+R)$ and $\sigma(A+R) \backslash \sigma_{w}(A+R)=p_{0}(A+R) \subseteq$ $\pi_{00}(A+R)$. Since, this is easily verified, $\sigma_{p}(A+R) \subseteq \bigcup_{i=1}^{n} \sigma_{p}\left(A_{i i}+R_{i i}\right)$ and $\pi_{00}\left(A_{i i}+R_{i i}\right)=\emptyset \Longrightarrow \pi_{00}(A+R)=\emptyset$, to complete the proof it would suffice to prove that the set $\pi_{00}\left(A_{i i}+R_{i i}\right)$ is empty. To this end, we start by observing from the commutativity of $A_{i i}$ and $R_{i i}$ that if $(0 \neq) x \in\left(A_{i i}+R_{i i}-\lambda\right)^{-1}(0)$ for some $\lambda \in \sigma\left(A_{i i}+R_{i i}\right)$, then $R_{i i}^{m} x \in\left(A_{i i}+R_{i i}-\lambda\right)^{-1}(0)$ for all $m \in \mathbb{N}$. Let $g(t)=\sum_{i=1}^{r} c_{i} t^{i}=c_{r} \prod_{i=1}^{r} t-\lambda_{i}$ be a polynomial such that $g\left(R_{i i}\right)=0$. Then, since the injectivity of $R$ implies that of $R_{i i}$ for all $1 \leq i \leq n, c_{r}=0$, and hence, by a finite induction argument, that $c_{i}=0$ for all $0 \leq i \leq r$. Consequently, $\left\{R_{i i}^{n} x\right\}$ is a linearly independent set of vectors in $\left(A_{i i}+R_{i i}-\lambda\right)^{-1}(0)$ and the eigenvalues of $A_{i i}+R_{i i}$ (and so also of $A_{i i}=A_{i i}+R_{i i}-R_{i i}$ ) have infinite multiplicity. Hence $\pi_{00}\left(A_{i i}+R_{i i}\right)=\emptyset$.

\section{Operators $A \in(H P)_{n}$ : Weyl's theorem}

For an upper triangular operator $A=\left(A_{i j}\right)_{1 \leq i, j \leq n} \in B\left(\mathcal{X}^{n}\right), A$ polaroid (even, hereditarily polaroid) does not imply $A_{i i}$ is hereditarily polaroid (even, polaroid) for all $1 \leq i \leq n$. For example, let $A=A_{11} \oplus A_{22}$, where $A_{11}=U \in$ $B\left(\ell^{2}\right)$ is the forward unilateral shift and $A_{22} \in B\left(\ell^{2}\right)$ is a non-nilpotent quasinilpotent operator. Then $\sigma(A)$ is the closed unit disc, $A$ is vacuously polaroid, and $A_{22}$ is not polaroid. Again, if $A=\left(\begin{array}{cc}U & 1-U U^{*} \\ 0 & U^{*}\end{array}\right)$, where $U \in B\left(\ell^{2}\right)$ is the forward unilateral shift, then $A$ is unitary (hence $(\mathcal{H P})), U \in(\mathcal{H P}), U^{*}$ is polaroid, but $U^{*} \notin(\mathcal{H} \mathcal{P})$. The following theorem considers the converse problem.

Theorem 3.1. Operators $A \in(H P)_{n}$ are polaroid.

Proof. Since $(\mathcal{H P})$ operators have SVEP $[6], \sigma(A)=\cup_{i=1}^{n} \sigma\left(A_{i i}\right)$. Hence

$$
\operatorname{iso} \sigma(A) \subseteq \bigcup_{i=1}^{n} \operatorname{iso} \sigma\left(A_{i i}\right) .
$$

Let $\lambda \in \operatorname{iso} \sigma(A)$. Then the polaroid hypothesis on $A_{i i}$ implies the existence of non-negative integers $p_{i}$ such that $H_{0}\left(A_{i i}-\lambda\right)=\left(A_{i i}-\lambda\right)^{-p_{i}}(0)$. (Evidently, $p_{j}=0$ whenever $\lambda \notin \sigma\left(A_{j j}\right)$.) We consider the operator $A_{2}=\left(\begin{array}{cc}A_{11} & A_{12} \\ 0 & A_{22}\end{array}\right)$ : the 
proof for the general case follows from a finitely repeated argument applied to $A_{j}=\left(\begin{array}{cc}A_{j-1} & * \\ 0 & A_{j j}\end{array}\right), 3 \leq j \leq n$. Let $p=\max \left\{p_{1}, p_{2}\right\}$. Then $H_{0}\left(A_{i i}-\lambda\right)=$ $\left(A_{i i}-\lambda\right)^{-p}(0) ; 1 \leq i \leq 2$. Observe that if $\left(\lambda \in \operatorname{iso} \sigma\left(A_{2}\right)\right.$ and $) x=x_{1} \oplus x_{2} \in$ $H_{0}\left(A_{2}-\lambda\right)$, then

$$
\begin{gathered}
\left\|\left(A_{2}-\lambda\right)^{m} x\right\|^{\frac{1}{m}}= \\
\|\left\{\left(A_{11}-\lambda\right)^{m} x_{1}+\sum_{j=0}^{m-1}\left(A_{11}-\lambda\right)^{m-j-1} A_{12}\left(A_{22}-\lambda\right)^{j} x_{2}\right\} \\
\oplus\left(A_{22}-\lambda\right)^{m} x_{2} \|^{\frac{1}{m}} \longrightarrow 0 \text { as } m \longrightarrow \infty
\end{gathered}
$$

implies that

$$
\lim _{m \longrightarrow \infty}\left\|\left(A_{22}-\lambda\right)^{m} x_{2}\right\|^{\frac{1}{m}}=0 \quad\left(\Longrightarrow x_{2} \in\left(A_{22}-\lambda\right)^{-p}(0)\right)
$$

and

$$
\lim _{m \longrightarrow \infty}\left\|\left(A_{11}-\lambda\right)^{m} x_{1}+\sum_{j=0}^{m-1}\left(A_{11}-\lambda\right)^{m-j-1} A_{12}\left(A_{22}-\lambda\right)^{j} x_{2}\right\|^{\frac{1}{m}}=0 .
$$

Choose $m=(t+1) p, t$ some positive integer, and set

$$
\left(A_{11}-\lambda\right)^{p} x_{1}+\sum_{j=0}^{p-1}\left(A_{11}-\lambda\right)^{p-j-1} A_{12}\left(A_{22}-\lambda\right)^{j} x_{2}=y .
$$

Then, since $\left(A_{22}-\lambda\right)^{r} x_{2}=0$ for integers $r \geq p$,

$$
\begin{aligned}
& \left(A_{11}-\lambda\right)^{m} x_{1}+\sum_{j=0}^{m-1}\left(A_{11}-\lambda\right)^{m-j-1} A_{12}\left(A_{22}-\lambda\right)^{j} x_{2} \\
= & \left(A_{11}-\lambda\right)^{(t+1) p} x_{1}+\sum_{j=0}^{(t+1) p-1}\left(A_{11}-\lambda\right)^{(t+1) p-j-1} A_{12}\left(A_{22}-\lambda\right)^{j} x_{2} \\
= & \left(A_{11}-\lambda\right)^{t p}\left\{\left(A_{11}-\lambda\right)^{p} x_{1}+\sum_{j=0}^{p-1}\left(A_{11}-\lambda\right)^{p-j-1} A_{12}\left(A_{22}-\lambda\right)^{j} x_{2}\right\} \\
= & \left(A_{11}-\lambda\right)^{t p} y .
\end{aligned}
$$

Since

$$
\left\|\left(A_{11}-\lambda\right)^{t p} y\right\|^{\frac{1}{t p}} \longrightarrow 0 \text { as } t \longrightarrow \infty
$$

$y \in H_{0}\left(A_{11}-\lambda\right)=\left(A_{11}-\lambda\right)^{-p}(0)$. Hence

$$
H_{0}\left(A_{2}-\lambda\right) \subseteq \oplus_{i=1}^{2}\left(A_{i i}-\lambda\right)^{-p}(0)=\left(A_{2}-\lambda\right)^{-p}(0) .
$$

Since $(T-\lambda)^{-p}(0) \subseteq H_{0}(T-\lambda)$ for every operator $T$ (and non-negative integer $p), H_{0}\left(A_{2}-\lambda\right)=\left(\bar{A}_{2}-\lambda\right)^{-p}(0)$.

To complete the proof, we now recall from above that if $\lambda \in \operatorname{iso} \sigma\left(A_{2}\right)$, then $\mathcal{X}^{2}=H_{0}\left(A_{2}-\lambda\right) \oplus K\left(A_{2}-\lambda\right)=\left(A_{2}-\lambda\right)^{-p}(0) \oplus\left(A_{2}-\lambda\right)^{p} \mathcal{X}^{2}$, i.e., $\lambda$ is a pole of order $\leq p$ of the resolvent of $A_{2}$. 
Recall, [1, p. 177], that a Banach space operator $A$ satisfies $a$-Weyl's theorem if $\sigma_{a}(A) \backslash \sigma_{a w}(A)=\pi_{00}^{a}(A)$, where $\pi_{00}^{a}(A)=\left\{\lambda \in\right.$ iso $\sigma_{a}(A): 0<\alpha(A-\lambda)<$ $\infty\}$. Let $H(\sigma(A))$ denote the class of functions $f$ which are analytic on a neighbourhood of $\sigma(A)$. For a polaroid operator with SVEP, $f(A)$ satisfies Weyl's theorem and $f\left(A^{*}\right)$ satisfies $a$-Weyl's theorem for every $f \in H(\sigma(A))$ [7, Lemma 3.1]. Hence:

Corollary 3.2. For operators $A \in(H P)_{n}, f(A)$ satisfies Weyl's theorem and $f\left(A^{*}\right)$ satisfies a-Weyl's theorem for every $f \in H(\sigma(A))$.

More is true, as we now proceed to prove. But before that some terminology. An operator $A$ is said to be algebraic if there exists a (non-trivial) polynomial $q(\cdot)$ such that $q(A)=0$. Banach space operators $F$ such that $F^{n}$ is finite dimensional for some positive integer $n$ are algebraic. We say that an operator $A$ is polynomially $(\mathcal{H P})$ if there exists a polynomial $g(\cdot)$ such that $g(A) \in(\mathcal{H P})$.

Theorem 3.3. Let $A \in \mathcal{A}$ be such that the operator $A_{0}=\bigoplus_{i=1}^{n} A_{i i}$ is a polynomially $(H P)_{n}$ operator. If $B=\bigoplus_{i=1}^{n} B_{i i} \in B\left(\mathcal{X}^{n}\right)$ is an algebraic operator which commutes $A_{0}$, then $f(A+B)$ satisfies Weyl's theorem and $f\left(A^{*}+B^{*}\right)$ satisfies a-Weyl's theorem for every $f \in H(\sigma(A+B))$.

Proof. The hypothesis that $A_{0}$ is polynomially $(H P)_{n}$ implies that $A_{i i}$ is polynomially $(\mathcal{H} \mathcal{P})$ for all $1 \leq i \leq n$. Hence $A_{i i}$ is polaroid and has SVEP for all $1 \leq i \leq n$ [7, Lemma 3.3]. Furthermore, since $B$ is algebraic and commutes with $A_{0}, B_{i i}$ is algebraic and commutes with $A_{i i}$ for all $1 \leq i \leq n$. Consequently, $A_{i i}+B_{i i}$ is polaroid and has SVEP for all $1 \leq i \leq n$. [7, Lemma 3.4]; hence $A+B$ has SVEP, a consequence of the fact that the diagonal elements $A_{i i}+B_{i i}$ of $A+B$ have SVEP, and $A+B$ is polaroid (by Theorem 3.1). This, by [7, Theorem 3.8], implies that $f(A+B)$ satisfies Weyl's theorem and $f\left(A^{*}+B^{*}\right)$ satisfies $a$-Weyl's theorem for every $f \in H(\sigma(A+B))$.

\section{References}

[1] P. Aiena, Fredholm and Local Spectral Theory with Applications to Multipliers, Kluwer, 2004.

[2] B. P. Duggal, Hereditarily normaloid operators, Extracta Math. 20 (2005), no. 2, 203217.

[3] _ Polaroid operators satisfying Weyl's theorem, Linear Algebra Appl. 414 (2006), no. $1,271-277$.

[4] _ Upper triangular operators with SVEP: spectral properties, Filomat 21 (2007), no. 1, 25-37.

[5] _ Weyl's theorem and hypercyclic/supercyclic operators, J. Math. Anal. Appl. 335 (2007), no. 2, 990-995.

[6] - Hereditarily polaroid operators, SVEP and Weyl's theorem, J. Math. Anal. Appl. 340 (2008), no. 1, 366-373.

[7] _ Polaroid operators, SVEP and perturbed Browder, Weyl theorems, Rend. Circ. Mat. Palermo (2) 56 (2007), no. 3, 317-330.

[8] B. P. Duggal, R. E. Harte, and I. H. Jeon, Polaroid operators and Weyl's theorem, Proc. Amer. Math. Soc. 132 (2004), no. 5, 1345-1349. 
[9] H. G. Heuser, Functional Analysis, John Wiley and Sons, 1982.

[10] I. B. Jung, E. Ko, and C. Pearcy, Spectral properties of some operator matrices, Arch. Math. (Basel) 80 (2003), no. 1, 37-46.

[11] K. B. Laursen and M. M. Neumann, Introduction to Local Spectral Theory, Clarendon Press, Oxford, 2000.

[12] M. Mbekhta, Généralisation de la décomposition de Kato aux opérateurs paranormaux et spectraux, Glasgow Math. J. 29 (1987), no. 2, 159-175.

[13] C. Pearcy, Some Recent Developments in Operator Theory, Regional Conference Series in Mathematics, No. 36. American Mathematical Society, Providence, R.I., 1978.

[14] V. Rakočević, Semi-Browder operators and perturbations, Studia Math. 122 (1997), no. $2,131-137$.

8 Redwood Grove, Northfield Avenue

EALing, London W5 4SZ, United Kingdom

E-mail address: bpduggal@yahoo.co.uk 